ARTICLE

DOI: $10.1038 / s 41467-017-01332-7$

\title{
Homeostatic plasticity shapes the visual system's first synapse
}

\author{
Robert E. Johnson ${ }^{1}$, Nai-Wen Tien ${ }^{1,2}$, Ning Shen ${ }^{1}$, James T. Pearson ${ }^{1,3}$, Florentina Soto ${ }^{1}$ \\ $\&$ Daniel Kerschensteiner ${ }^{1,4,5,6}$
}

Vision in dim light depends on synapses between rods and rod bipolar cells (RBCs). Here, we find that these synapses exist in multiple configurations, in which single release sites of rods are apposed by one to three postsynaptic densities (PSDs). Single RBCs often form multiple PSDs with one rod; and neighboring RBCs share $13 \%$ of their inputs. Rod-RBC synapses develop while $\sim 7 \%$ of RBCs undergo programmed cell death (PCD). Although PCD is common throughout the nervous system, its influences on circuit development and function are not well understood. We generate mice in which $\sim 53$ and $\sim 93 \%$ of RBCs, respectively, are removed during development. In these mice, dendrites of the remaining RBCs expand in graded fashion independent of light-evoked input. As RBC dendrites expand, they form fewer multi-PSD contacts with rods. Electrophysiological recordings indicate that this homeostatic co-regulation of neurite and synapse development preserves retinal function in dim light.

\footnotetext{
${ }^{1}$ Department of Ophthalmology and Visual Sciences, Washington University School of Medicine, Saint Louis, MO 63110, USA. ${ }^{2}$ Graduate Program in Neuroscience, Washington University School of Medicine, Saint Louis, MO 63110, USA. ${ }^{3}$ Graduate Program in Developmental, Regenerative and Stem Cell Biology, Washington University School of Medicine, Saint Louis, MO 63110, USA. ${ }^{4}$ Department of Neuroscience, Washington University School of Medicine, Saint Louis, MO 63110, USA. ${ }^{5}$ Department of Biomedical Engineering, Washington University School of Medicine, Saint Louis, MO 63110, USA. ${ }^{6}$ Hope Center for Neurological Disorders, Washington University School of Medicine, Saint Louis, MO 63110, USA. Correspondence and requests for materials should be addressed to D.K. (email: kerschensteinerd@wustl.edu)
} 
T he ability of mammals to see in low light depends on the synapses between rods and rod bipolar cells (RBCs) ${ }^{1}$. Mutations in genes involved in the formation and function of these synapses cause congenital stationary night blindness $(\mathrm{CSNB})$ in people ${ }^{2}$. Key molecular events in rod-RBC synapse assembly have been uncovered using mouse models of CSNB and other strategies ${ }^{3-7}$. A recent electron microscopy study showed that the spherical rod axon terminals (i.e., rod spherules) connect to varying numbers of $\mathrm{RBC}$ dendrites ${ }^{8}$, suggesting that rod-RBC synapse configurations might be malleable within molecularly defined boundaries. However, because only a few RBCs were reconstructed $^{8}$, the range of configurations of rod-RBC synapses remains uncertain, and whether plasticity controls their distribution has not been tested.

Developmental plasticity is essential for the emergence of precise circuits; and its dysregulation underlies common neurodevelopmental disorders ${ }^{9}, 10$. Known plasticity mechanisms include axon and dendrite remodeling ${ }^{11-13}$, synapse formation and elimination ${ }^{14-18}$, and changes in the geometry and molecular architecture of synapses ${ }^{19-21}$. In developing circuits, populations of same-type neurons need to coordinate their connectivity to homogeneously cover input and target cell types, while individual neurons need to adjust their connectivity to avoid saturation and quiescence. Because most studies so far have focused on individual plasticity mechanisms and their underlying signals ${ }^{22-24}$, how different plasticity mechanisms (e.g., neurite remodeling and synapse formation) are co-regulated during development to optimize wiring of neuronal populations and individuals in vivo is unknown.

Throughout the developing nervous system, many neurons undergo programmed cell death (PCD), adjusting the complement and density of neuronal populations in emerging circuits $^{25,26}$. PCD triggers plasticity in the remaining neurons, which take over innervation of vacated inputs and targets. The retina is an ideal system for studying cell density-dependent plasticity, because axons and dendrites of each cell type cover synaptic layers uniformly ${ }^{27,28}$. Cell density-dependent plasticity has been shown to regulate axon and dendrite growth of some retinal neurons ${ }^{29,30}$ but not others ${ }^{31,32}$. To what extent RBC axons and dendrites undergo cell density-dependent plasticity is incompletely understood $^{33}$, and how cell density-dependent plasticity regulates synaptic development of any neuron is unknown.

To analyze the influence of cell density-dependent plasticity on RBC development and retinal circuit function, we generated mice in which $\sim 53$ and $\sim 93 \%$ of RBCs, respectively, are removed by transgenic expression of diphtheria toxin concurrent with naturally occurring $\mathrm{PCD}^{26}$. We find that dendritic and axonal territories of the remaining RBCs increase in graded fashion to improve population coverage, whereas multi-PSD synapses on dendrites and synapse density of axons are reduced to restrain connectivity of individual RBCs. This coordinated plasticity of neurites and synapses occurs independent of light-evoked input from rods and preserves retinal output in dim light.
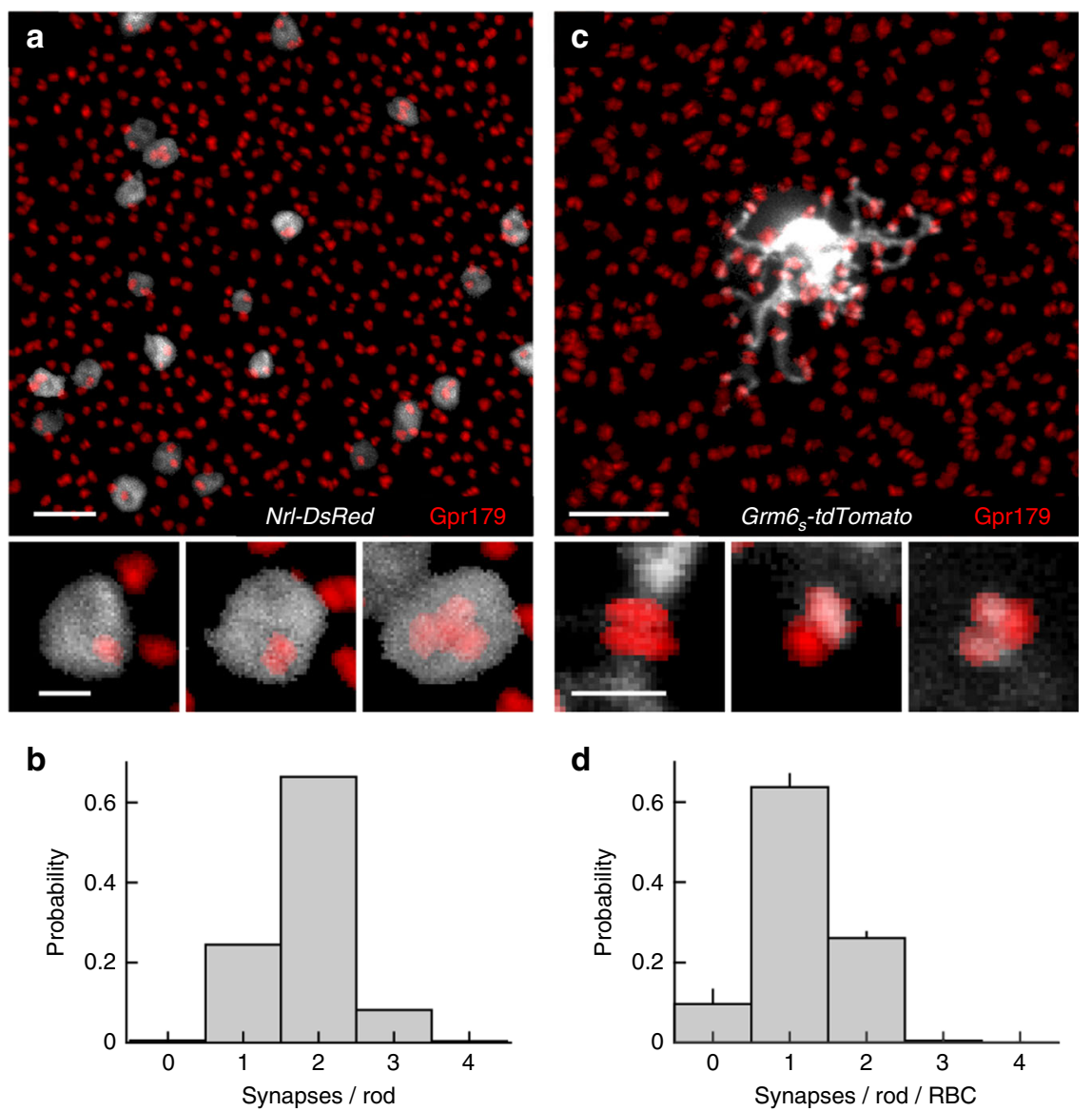

Fig. 1 Rod-RBC synapses exist in different configurations. a Maximum intensity projection of a confocal image stack of the outer plexiform layer of a retina, in which rods were electroporated with $\mathrm{Nrl}$-DsRed (gray) and in which RBC postsynaptic sites were stained for Gpr179 (red). Scale bar represents $5 \mu$ m. The top panel shows an overview, whereas the bottom panels present higher magnification views of individual rod spherules. $\mathbf{b}$ Histogram of the number of Gpr179 clusters per rod observed in our data ( $n=555$ rods, $n=12$ mice). Scale bar represents $0.5 \mu$ m. c Analogous to a, but showing AAV-mediated (Grm6s-tdTomato) labeling of an individual RBC in gray. For visual clarity, the RBC and rod synapses were digitally isolated in Amira. d Population data (mean \pm SEM) showing the distribution of postsynaptic densities assembled by each RBC per rod spherule $(n=29$ RBCs, $n=8$ mice) 

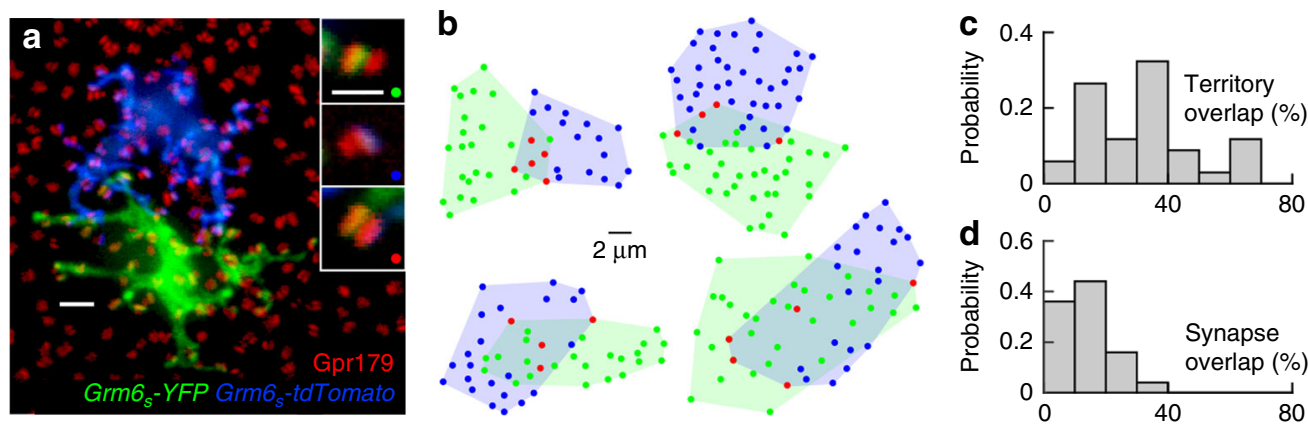

Fig. 2 RBC dendrites overlap and share rod input. a Maximum intensity projection of a confocal image stack of two adjacent RBCs labeled with spectrally separable fluorophores via AAVs (Grm6s - tdTomato in blue and $\mathrm{Grm}_{s}-$ YFP in green). RBC postsynaptic sites are stained for Gpr179 (red). For visual clarity, the two RBCs were digitally isolated in Amira. Scale bar represents $2 \mu \mathrm{m}$. Insets on the right show higher magnification views of Gpr179 clusters contacted by either (top two panels) or both (bottom panel) RBCs. Scale bar represents $1 \mu \mathrm{m}$. $\mathbf{b}$ Representative examples of dendritic and synaptic overlap of four pairs of RBCs. Shaded areas represent dendritic territories. Rods targeted by dendrites of either RBC are marked by green and blue circles; rods targeted by dendrites of both RBCs are indicated by red circles. $\mathbf{c}$, $\mathbf{d}$ Distributions of dendritic territory overlap $(\mathbf{c}, n=37$ pairs, $n=10$ mice) and synaptic overlap (d, $n=$ 28 pairs, $n=8$ mice) between neighboring RBCs
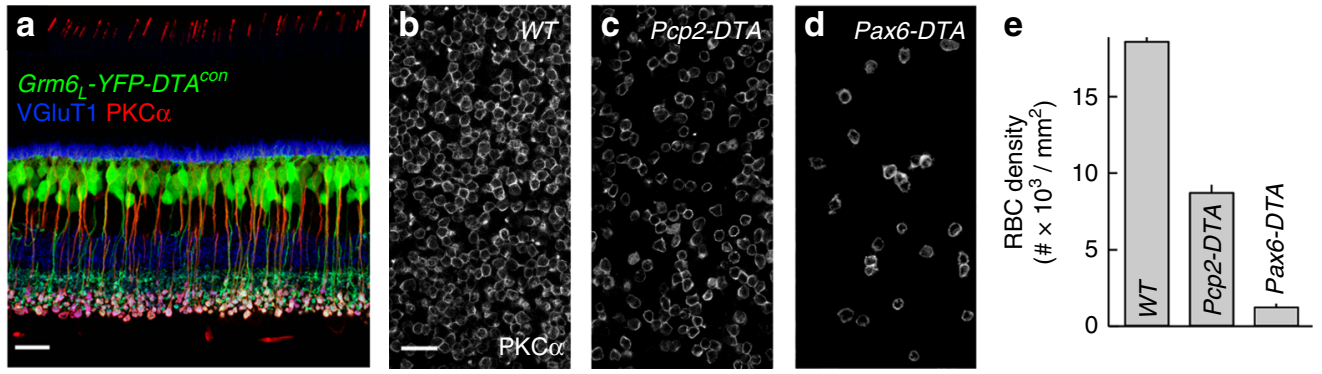

Fig. 3 Transgenic removal of RBCs from developing circuits. a Section through a Grm6L-YFP-DTA ${ }^{\text {con }}$ retina in which ON bipolar cells express YFP (green) stained for VGluT1 (blue), which labels photoreceptor and bipolar cell axon terminals, and for the RBC-specific marker PKC $\alpha$ (red). Scale bar indicates 20 $\mu \mathrm{m}$. b-d Retinal flat mounts from wild-type (b), Pcp2-DTA (c), and Pax6-DTA (d) mice stained for PKC $\alpha$. Scale bar indicates $20 \mu \mathrm{m}$. e Summary data (mean \pm SEM) of RBC densities in wild-type $(n=23$ mice), Pcp2-DTA $(n=8$ mice), and Pax6-DTA $(n=22$ mice) mice. By Kruskal-Wallis one-way ANOVA testing, the density of RBCs was lower in Pcp2-DTA and Pax6-DTA compared to wild-type retinas $\left(p<0.04\right.$ and $p<10^{-8}$, respectively), and lower in Pax6DTA than in Pcp2-DTA retinas $(p<0.049)$

\section{Results}

Rod-RBC synapses exist in different configurations. To examine the configurations of rod-RBC synapses, we first sparsely and selectively labeled rods by in vivo electroporation of a plasmid in which the fluorescent protein DsRed is expressed from promoter elements of the rod-specific neural retina leucine zipper $(\mathrm{Nrl})$ transcription factor (Fig. 1a, Nrl-DsRed) ${ }^{34,35}$. Each rod contains a single presynaptic ribbon ${ }^{36,37}$. By contrast, we observed a range of RBC PSDs containing the probable G-protein coupled receptor 179 (Gpr179) ${ }^{38-40}$ in individual rod spherules (from one to three receptor clusters in $>99 \%$ of spherules) (Fig. 1a, b). The distribution of RBC postsynaptic specializations per rod was similar when we stained for the metabotropic glutamate receptor mGluR6 $6^{3,41}$ instead of Gpr179, and when super-resolution rather than conventional confocal microscopy was used (Supplementary Fig. 1). We next explored how individual RBCs connect with rods in their dendritic fields. We generated adeno-associated viruses (AAVs) that expressed the fluorescent protein tdTomato from promoter elements of the Grm6 gene, which encodes mGluR6 $\left(\mathrm{Grm}_{\mathrm{S}} \text {-tdTomato }\right)^{42}$. Intravitreal injections of Grm6 $6_{S}$-tdTomato labeled ON bipolar cells, which include RBCs and ON cone bipolar cells. RBCs could easily be identified by their characteristic morphology ${ }^{15}, 43$. We flat-mounted retinas of mice injected with $G r m 6_{S}$-tdTomato and stained synaptic contacts for Gpr179 (Fig. 1c). Rod labeling had shown that overlapping Gpr179 clusters were invariably localized within the same spherule (Fig. 1a). We therefore counted overlapping Gpr179 clusters as synapses with a single rod, and determined whether a given cluster co-localized with a dendritic tip of the labeled RBC. We found that on average RBCs fail to be innervated $\sim 10 \%$ of rods in their dendritic fields, assemble a single PSD in $\sim 63 \%$ of spherules, and form multi-PSD contacts with $\sim 27 \%$ of rods (Fig. 1c, d). Rod-RBC synapses thus exist in different configurations, in which a single presynaptic release site is apposed by one to three PSDs belonging to one or more RBCs.

Dendrites of neighboring RBCs overlap and share rod input. To visualize dendritic interactions of neighboring RBCs we labeled cells with spectrally separable fluorescent proteins via two AAVs $\left(\right.$ Grm $_{S^{-}}$tdTomato and $\left.\mathrm{Grm}_{S_{S^{-}}} Y F P\right)$ and stained retinas for Gpr179 (Fig. 2a). We restricted our analysis to RBC pairs whose somata were $<10 \mu \mathrm{m}$ apart (center-center distance). Dendritic territories of these RBC pairs, defined as the smallest convex polygons encompassing all Gpr179-bearing dendritic tips, overlapped on average by $30 \%$, with relatively large variability in the amount of overlap between pairs (coefficient of variation: $62 \%$; Fig. 2b, c). By comparison, RBC pairs shared a smaller (13\%) fraction of input from rods, and the variability in the fraction of input shared between RBC pairs was lower (coefficient of variation: $50 \%$; Fig. $2 \mathrm{~b}, \mathrm{~d}$ ) than that of their dendritic overlap.

Transgenic removal of RBCs from developing circuits. To probe the influence of cell density-dependent plasticity on neurite 

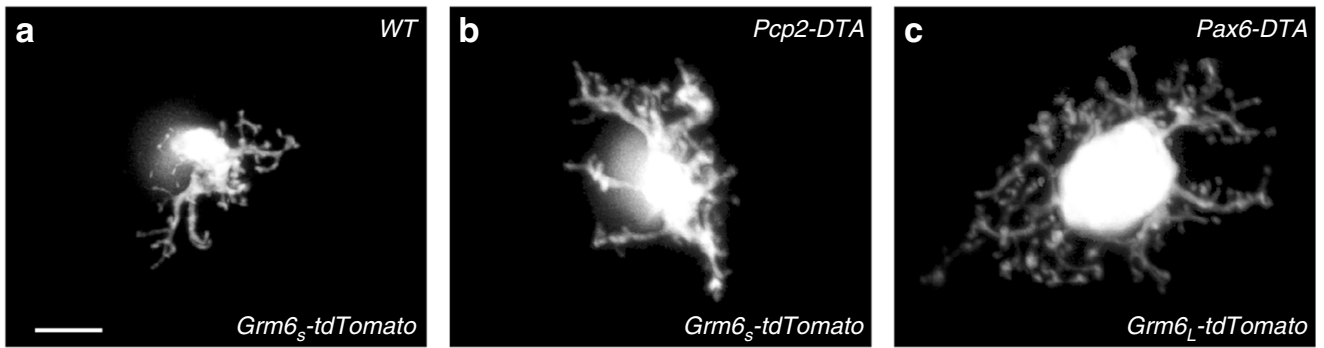

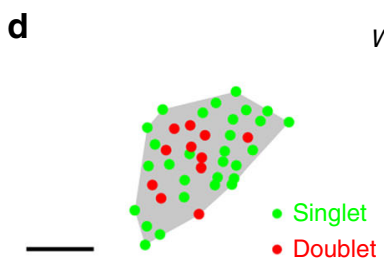

e

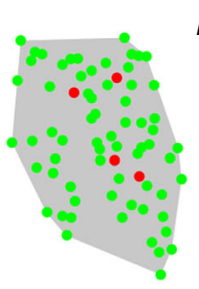

g
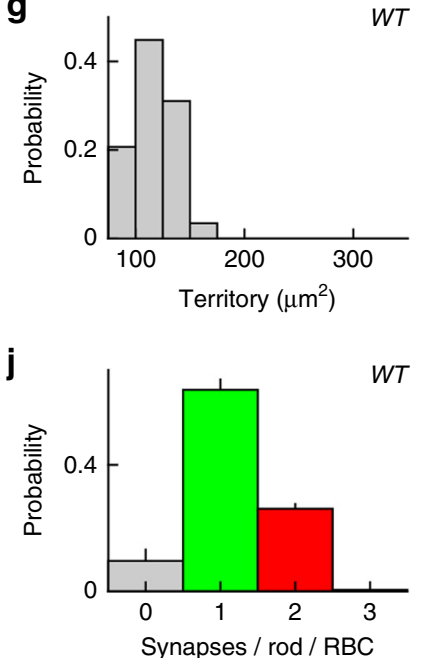

WT
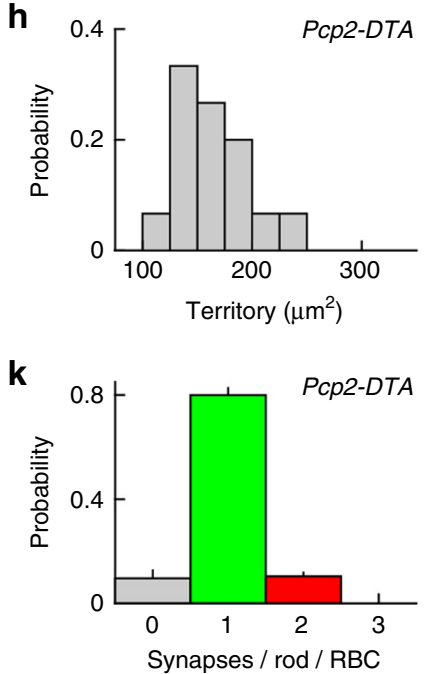

Pcp2-DTA
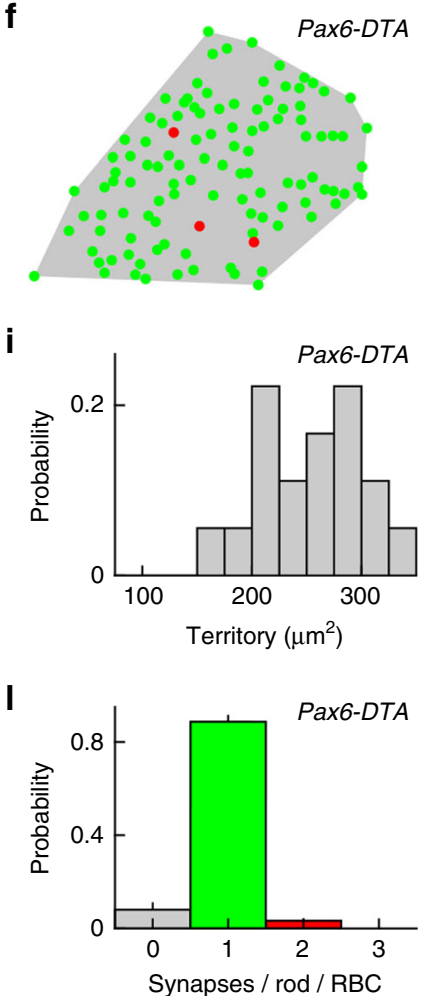

Fig. 4 Cell density regulates RBC dendrite and synapse development. a-c Maximum intensity projection of dendritic trees of individual RBCs labeled via AAVs (Grm6s-tdTomato) or in a transgenic line (Grm6 L-tdTomato) in wild-type (a), Pcp2-DTA (b), and Pax6-DTA (c) mice. For visual clarity, RBCs were digitally isolated in Amira. Scale bar indicates $5 \mu \mathrm{m}$. d-f Maps of dendritic territories (gray shaded areas) and synapse configurations (singlets: green circles, doublets: red circles) of the cells shown in a-c. Scale bar indicates $5 \mu \mathrm{m}$. $\mathbf{g}$-i Summary data of RBC dendritic territories in wild-type (g, $n=29$ RBCs, $n=8$ mice), Pcp2-DTA ( $h, n=15$ RBCs, $n=6$ mice), and Pax6-DTA (i, $n=18$ RBCs, $n=6$ mice) mice. By Kruskal-Wallis one-way ANOVA testing, RBC dendrite territories in Pcp2-DTA and Pax6-DTA retinas were larger than in wild-type retinas $\left(p<0.005\right.$ and $p<10^{-8}$, respectively), and RBC dendrite territories were larger in Pax6-DTA than in Pcp2-DTA retinas $(p<0.02)$. $\mathbf{j}-\mathbf{I}$ Population data (mean $\pm \mathrm{SEM}$ ) of the distribution of synapses configurations on RBC dendrites in wild-type ( $\mathbf{j}, n=29$ RBCs, $n=8$ mice), Pcp2-DTA ( $\mathbf{k}, n=15$ RBCs, $n=6$ mice), and Pax6-DTA (I, $n=10$ RBCs, $n=4$ mice) retinas. By Kruskal-Wallis one-way ANOVA testing, the average number of PSDs per rod and RBC was lower in Pax6-DTA than in wild-type retinas $(p<0.003)$

and synapse development, we generated mice that conditionally express an attenuated version of diphtheria toxin ${ }^{44}$ in RBCs and ON cone bipolar cells $\left(G r m 6_{L}-Y F P-D T A^{c o n}\right.$ mice ${ }^{16}$, Fig. 3a). We crossed $\mathrm{Grm6}_{L^{-}}$YFP-DTA ${ }^{\text {con }}$ mice to Pcp2-Cre ${ }^{45}$ or Pax6-Cre ${ }^{46}$ lines to produce $P c p 2-D T A$ and Pax6-DTA mice, respectively. In Pcp2-Cre mice, Cre recombinase is expressed in $\sim 50 \%$ of RBCs and in a small subset of photoreceptors (Supplementary Fig. 2a-d). Accordingly, cell removal in Pcp2-DTA mice is restricted to RBCs, whose density is reduced by $\sim 53 \%$ (Fig. $3 b, c$, e). In Pax6-Cre mice, Cre is expressed in retinal progenitor cells and recombination therefore occurs in all retinal cell types. Cre expression in these mice is region specific and excludes a dorsoventral wedge through the center of the retina (Supplementary Fig. 2e $)^{46}$. In the Cre-positive regions of Pax6-DTA retinas, $\sim 93 \%$ of RBCs are removed without microglial activation (Fig. $3 \mathrm{~b}, \mathrm{~d}$, e; Supplementary Fig. 3). In addition to RBCs, ON cone bipolar cells in the Cre-positive regions are deleted to varying degrees ${ }^{47}$. By staining for the cell type-specific marker PKC $\alpha$ at different postnatal ages, we found that RBCs in Pax6-DTA mice are removed around postnatal day 9 (P9: 80\%<WT, P15: $93 \%<\mathrm{WT})$. Thus, we have generated mice (Pcp2-DTA and Pax6$D T A)$, in which distinct fractions of RBCs are removed from circuits concurrent with developmental $\mathrm{PCD}^{26}$.

Cell density regulates $\mathrm{RBC}$ dendrite and synapse development. We used Pcp2-DTA and Pax6-DTA mice to study the influence of cell density-dependent plasticity on RBC dendrites and rod-RBC synapses. RBCs were labeled using either a transgenic line (Grm6 $6_{L}$-tdTomato) or AAVs (Grm6 $6_{S}$-tdTomato). RBC morphologies were indistinguishable between these labeling strategies and results from both approaches were therefore combined. Comparisons of wild-type, Pcp2-DTA, and Pax6-DTA retinas revealed that $\mathrm{RBC}$ dendrites expand in graded fashion as the density of 
a
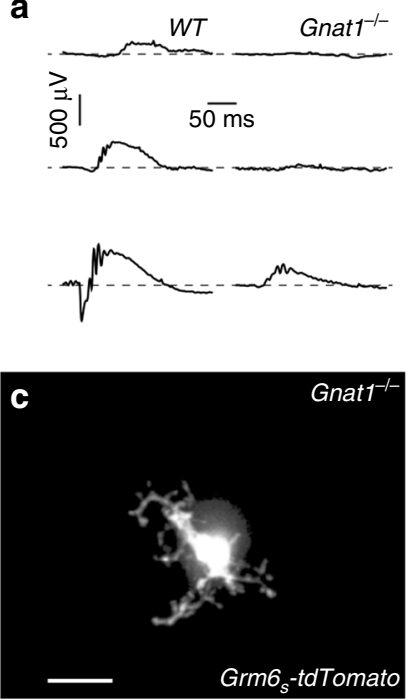

e

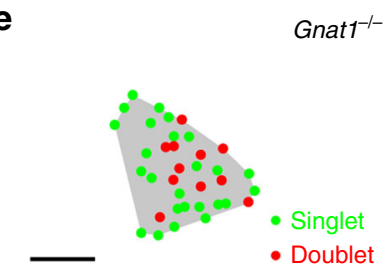

9
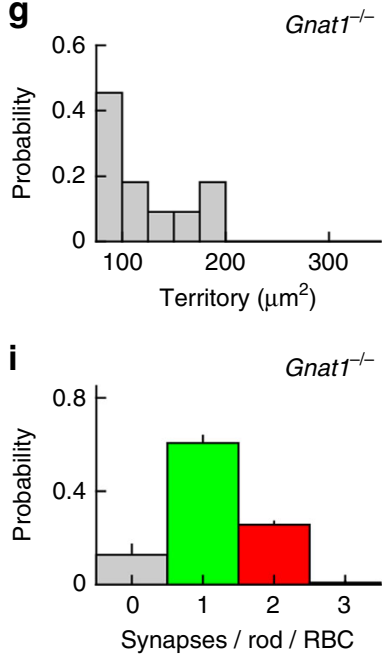

b
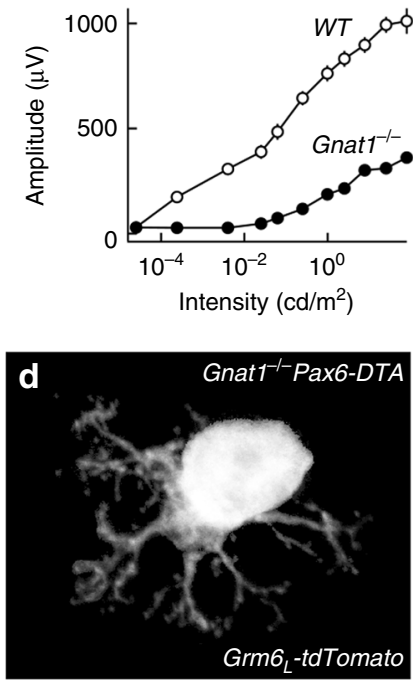

f

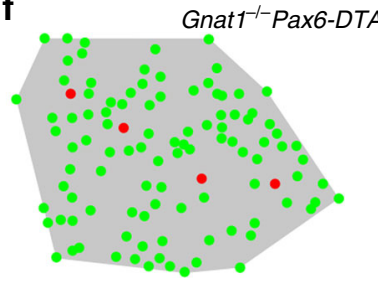

h

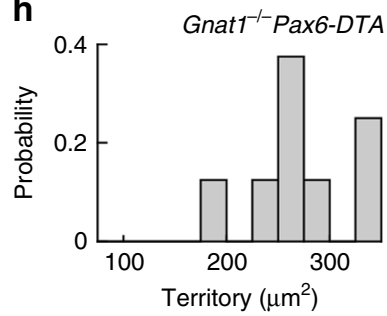

j

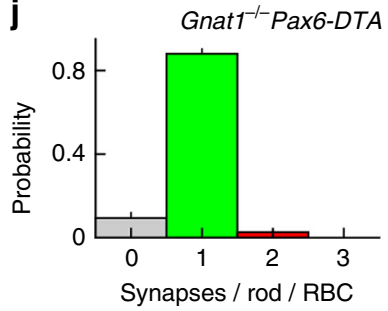

Fig. 5 RBC plasticity is independent of light-evoked rod input. a Representative ERG responses to stimuli of increasing light intensity (top row: $0.00025 \mathrm{~cd} / \mathrm{m}^{2}$, middle row: $0.25 \mathrm{~cd} / \mathrm{m}^{2}$, bottom row: $25 \mathrm{~cd} / \mathrm{m}^{2}$ ) recorded from wild-type (left column) and $\mathrm{Gnat}^{-/-}$(right column) retinas. b Summary data of intensity response functions measured from b-wave amplitudes in wild-type (open circles, $n=6$ mice) and $\mathrm{Gnat}^{-1-}$ (filled circles, $n=4$ mice) animals. c, d MIP of dendritic trees of individual RBCs labeled via AAVs (Grm6s - tdTomato) or in a transgenic line (Grm6 $\sigma_{L^{-}}$ tdTomato) in Gnat1 ${ }^{-1-}$ (c) and Gnat1-/- Pax6-DTA (d) mice. For visual clarity, RBCs were digitally isolated in Amira. Scale bar indicates $5 \mu \mathrm{m}$. e, $\mathbf{f}$ Maps of dendritic territories (gray shaded areas) and synapse configurations (singlets: green circles, doublets: red circles) of the cells shown in $\mathbf{c}$ and $\mathbf{d}$. Scale bar indicates $5 \mu \mathrm{m} . \mathbf{g}$, $\mathbf{h}$ Summary data of RBC dendritic territories in Gnat ${ }^{-1-}(\mathbf{g}, n=13 \mathrm{RBC}, n=5$ mice $)$ and $\mathrm{Gnat}^{-1-}$ Pax6-DTA (i, $n=8$ RBCs, $n=4$ mice, $p<0.001)$ mice. $\mathbf{i}, \mathbf{j}$ Population data (mean \pm SEM) of the distribution of synapses configurations on RBC dendrites in Gnat1 ${ }^{-/-}(\mathbf{i}, n=11 \mathrm{RBCs})$ and Gnat1 ${ }^{-/-}$Pax6-DTA $(\mathbf{j}, n=8$ RBCs, $n=4$ mice, $p<0.04)$ retinas

RBCs is reduced (Fig. 4a-i). To determine whether dendrite growth is regulated by local (e.g., contact-mediated) or global (e.g., long-range diffusible messenger) signals, we analyzed the morphology of RBCs at the border of Cre-positive and Cre-negative regions in Pax6-DTA retinas. There, we frequently observed clusters of RBCs surrounded by RBC-depleted areas. The dendrites of RBCs in such clusters invariably extend away from remaining neighbors into the depleted areas (Supplementary Fig. 4). This suggests that, similar to other retinal neurons ${ }^{29,30}$, the growth of RBC dendrites is constrained by local homotypic signals.

To test whether local interactions are required to maintain $\mathrm{RBC}$ dendrite size in the adult retina, we crossed Pcp2-Cre mice to a transgenic strain in which the diphtheria toxin receptor is expressed in a Cre-dependent manner (DTR mice) ${ }^{48}$. However, diphtheria toxin injections that completely remove other retinal cells targeted with this strategy 49,50 caused only a minor reduction in RBC density in double-positive offspring (Pcp2-DTR, Supplementary Fig. 5). We therefore could not analyze the extent of cell density-dependent plasticity in the adult retina.

The density of rods is unchanged in Pcp2-DTA and Pax6-DTA mice (Supplementary Fig. 6), and as RBC dendrites in their retinas expand, they contact an increasing number of rods (Fig. 4a-f). This improves input coverage by the remaining RBC population, but carries the risk of saturating input to individual cells. Interestingly, analysis of rod-RBC synapses revealed that whereas RBCs in wild-type retinas form two (i.e., doublets) or more PSDs with $27 \%$ of rods, the frequency of PSD doublets is gradually reduced in Pcp2-DTA (10\%) and Pax6-DTA (3\%) retinas. This homeostatic shift from doublet to singlet (i.e., one PSD with one rod spherule) synapses could serve to limit input to expanded dendrites.

RBC plasticity is independent of light-evoked rod input. We next tested to what extent the remodeling of dendrites and synapses elicited by changes in RBC density is regulated by input from rods. In mice lacking rod transducin- $\alpha$ (Gnat $1^{-/-}$mice), rods fail to respond to light and scotopic ERG responses are suppressed (Fig. 5a, b) ${ }^{51}$. We found that RBC dendrites in Gnat 1 $-/-$ mice occupy normal territories and develop synapses with configurations similar to those observed in wild-type retinas (Fig. 5c, e, g, i). Moreover, in Gnat1 ${ }^{-/-}$Pax6-DTA mice, RBC dendrites expand and shift from doublet to singlet synapses as they do in Pax6-DTA mice (Fig. 5d, f, h, j). Thus, dendrite and synapse development, and cell density-dependent plasticity of RBCs appear to be independent of light-evoked input from rods.

To silence spontaneous transmitter release from rods, we crossed mice in which the light chain of tetanus toxin (TeNT) is expressed in a Cre-dependent manner (TeNT mice $)^{52}$ to Rhodopsin-iCre mice, which express a codon-improved version of Cre recombinase (iCre $)^{53}$, under control of the rod-specific rhodopsin promoter $^{54}$. Unfortunately, double-positive offspring (Rhodopsin-TeNT) showed rapid photoreceptor degeneration (Supplementary Fig. 7). We were therefore unable to analyze the influence of spontaneous transmitter release from rods on $\mathrm{RBC}$ dendrite and synapse development and plasticity.

Cell density regulates $\mathrm{RBC}$ axon and synapse development. Given the homeostatic plasticity of RBC dendrites and rod-RBC connections, we next tested whether cell density similarly co-regulates the development of RBC axons and their synapses. We found that $\mathrm{RBC}$ axons expand in graded fashion as the density of RBCs around them decreases in Pcp2-DTA and Pax6-DTA mice (Fig. 6a-f). Labeling for C-terminal binding protein 2 , a component of presynaptic ribbons ${ }^{55}$, then 

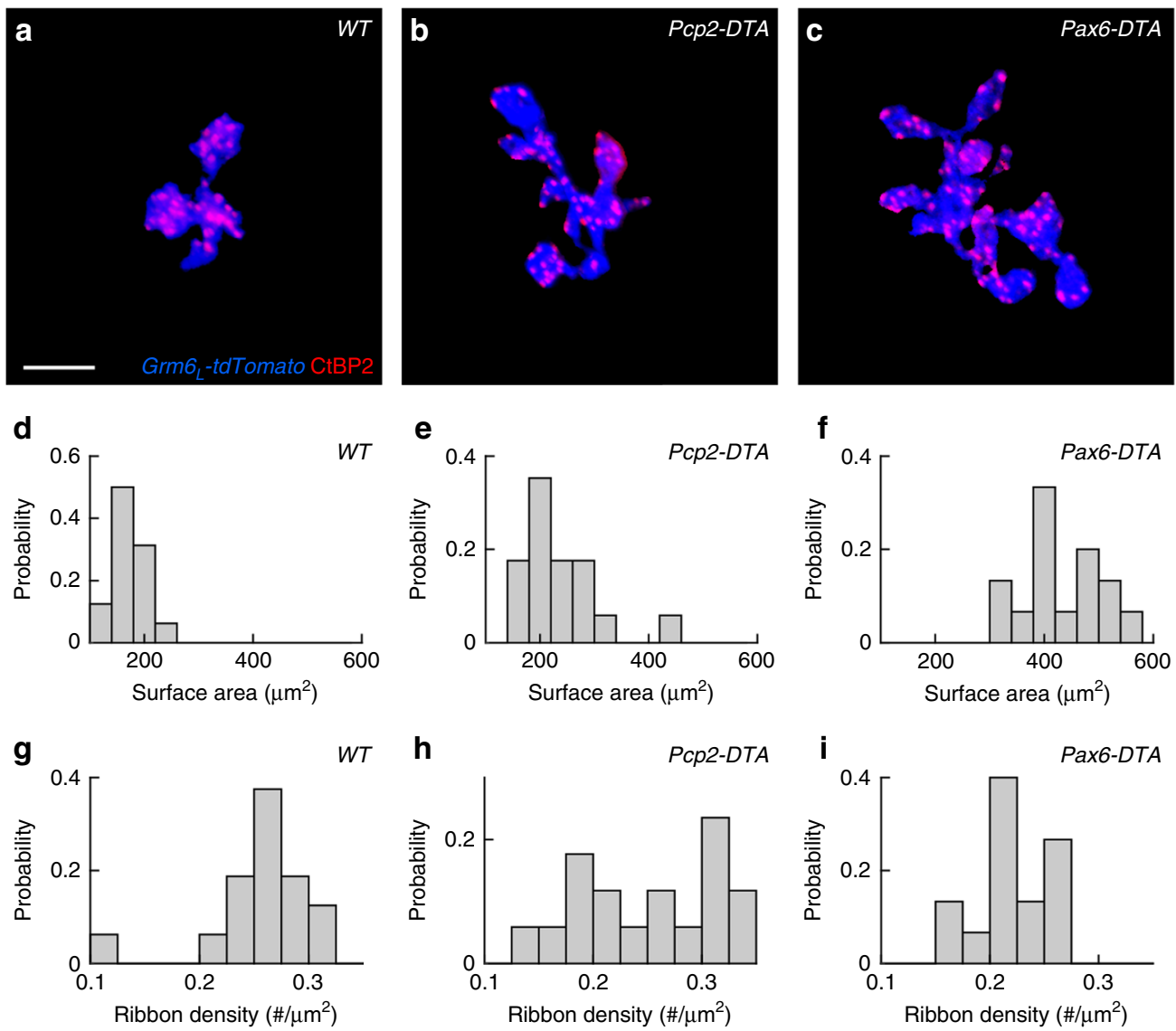

Fig. 6 Cell density regulates RBC axon and synapse development. a-c Maximum intensity projection of RBC axon terminals labeled by transgenic expression of tdTomato (Grm6 $6_{\mathrm{L}}$-tdTomato in blue) and presynaptic release sites stained for the C-terminal binding protein 2 (CtBP2, red) in wild-type (a), Pcp2-DTA (b), and Pax6-DTA (c) retinas. For visual clarity, RBC axons and their synapses were digitally isolated in Amira. Scale bar indicates $5 \mu \mathrm{m}$. d-f Summary data of RBC axon surface areas in wild-type (d, $n=16$ RBCs), Pcp2-DTA (e, $n=17$ RBCs), and Pax6-DTA (f, $n=15$ RBCs) mice. By Kruskal-Wallis one-way ANOVA testing, RBC axon surface areas in Pcp2-DTA and Pax6-DTA retinas were greater than in wild-type retinas ( $p<0.02$ and $p<10^{-8}$, respectively), and RBC axon surface areas were greater in Pax6-DTA than in Pcp2-DTA retinas $(p<0.003)$. g-i Population data (mean \pm SEM) from wild-type ( $\mathbf{g}, n=16 \mathrm{RBCs}$ ), Pcp2-DTA ( $\mathbf{h}, n=17 \mathrm{RBCs}$ ), and Pax6-DTA (i, $n=15 \mathrm{RBCs}$ ) retinas show that synapse density tends to decrease with increasing axon size. By Kruskal-Wallis one-way ANOVA testing, RBC synapse densities in Pcp2-DTA and Pax6-DTA retinas were lower than in wild-type retinas $(p<0.003$ and $p<0.02$, respectively)

showed that the density of RBC output synapses is reduced in Pcp2-DTA and even further in Pax6-DTA retinas (Fig. 6g-i). Thus, cell density-dependent plasticity co-regulates axon size and synapse density in seemingly homeostatic fashion, similar to the changes observed in RBC dendrites and rod-RBC synapse configurations.

RBC plasticity preserves retinal output in dim light. We hypothesized that the homeostatic co-regulation of neurites and synapses of RBC dendrites and axons serves to improve input and target coverage by the remaining population of RBCs, while maintaining manageable input and output connectivity for individual neurons; and that this in turn preserves retinal function in dim light. To test this hypothesis, we recorded the synaptic input and spike responses of $\mathrm{ON} \alpha$ retinal ganglion cells (ON $\alpha-\mathrm{RGCs})$, which are sensitive to small changes in luminance (i.e., low contrast) even in dim light ${ }^{56}$. In Cre-positive regions of Pax6$D T A$ mice, few RBCs are left and varying fractions of cone bipolar cells are deleted. We therefore focused on Pcp2-DTA retinas, in which $\sim 53 \%$ of RBCs are selectively removed. Whole-cell patch clamp recordings revealed that in spite of this loss, all ONa-RGCs (11 of 11 cells) responded to stimuli at light levels preferentially activating the rod bipolar pathway (Supplementary Fig. 8). Moreover, the amplitudes of excitatory inputs and spike responses of $\mathrm{ON} \alpha$-RGCs were unchanged in Pcp2-DTA compared to wild-type retinas (Fig. $7 \mathrm{a}, \mathrm{b}, \mathrm{d}, \mathrm{e}$ ), and the characteristically linear contrast response functions of $\mathrm{ON} \alpha$-RGCs were preserved in Pcp2-DTA mice (Fig. 7c, f).

RGC types differ in their spatiotemporal receptive fields. To test whether RGC type-specific receptive field properties are altered in Pcp2-DTA mice, we recorded spike trains of ON $\alpha$-RGCs and OFF $\alpha$-RGCs during presentation of circular white noise stimuli. In these stimuli, the intensities of rings of equal area centered on the recorded cell were chosen at random every $33 \mathrm{~ms}$ (refresh rate: $30 \mathrm{~Hz}$ ) from a Gaussian distribution. We then mapped receptive fields by spike-triggered stimulus averaging 57,58 . Receptive field maps of ON $\alpha$-RGCs and OFF $\alpha$ RGCs were indistinguishable between wild-type and Pcp2-DTA mice (Fig. 8a-c). Thus, in addition to contrast coding, cell typespecific spatiotemporal filtering of visual signals is preserved in Pcp2-DTA mice, supporting the notion that cell densitydependent plasticity co-regulates neurite and synapse development of RBCs to preserve retinal function in dim light.

\section{Discussion}

A recent electron microscopy study reconstructed the connectivity patterns of eight RBCs in mice, and found that signals from one rod often diverge to multiple RBCs and that one RBC 
a

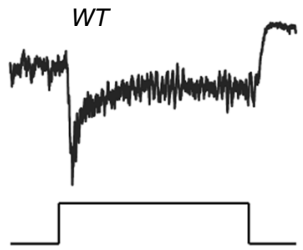

d

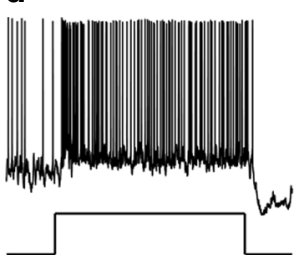

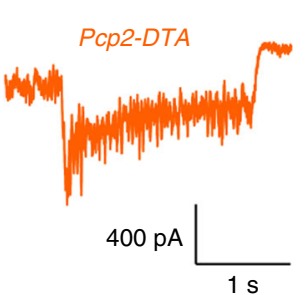

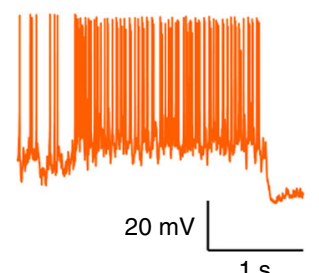

b

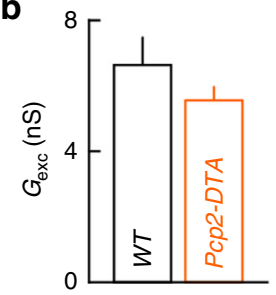

e

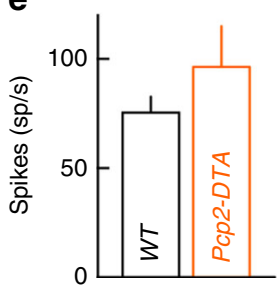

c

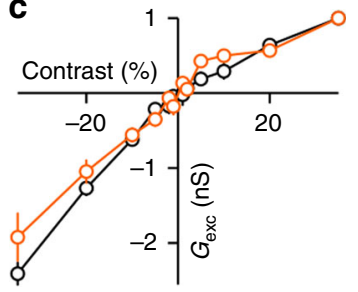

f

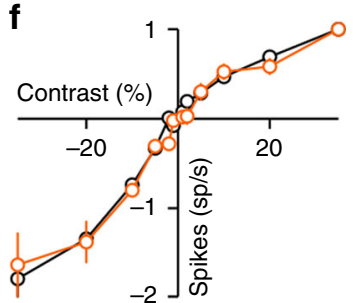

Fig. 7 Excitatory input and spike responses of ON $\alpha-R G C s$ in wild-type and Pcp2-DTA mice. a EPSC responses to dim light steps (three rhodopsin isomerization/rod/s, $3 \mathrm{R}^{\star}$ ) recorded from ON $\alpha-R G C s$ in wild-type (left, black) and Pcp2-DTA (right, orange) retinas. b Summary data (mean \pm SEM) of the excitatory conductances elicited by dim light steps (as shown in $\mathbf{a}$ ) in ON $\alpha-R G C$ of wild-type (black, $n=7$ cells, $n=2$ mice) and Pcp2-DTA (orange, $n=6$ cells, $n=2$ mice, $p>0.5$ by Wilcoxon rank sum test) mice. c Population data for contrast response functions of excitatory conductances of ON $\alpha$-RGCs recorded in wild-type (black, $n=6$ cells, $n=2$ mice) and Pcp2-DTA (orange, $n=3$ cells, $n=2$ mice, $p>0.1$ by bootstrapping methods) retinas. $\mathbf{d}-\mathbf{f}$ Analogous to $\mathbf{a}-\mathbf{c}$, but for spike responses instead of excitatory inputs. Responses to dim light steps (wild type $n=9$ cells, $n=2$ mice Pcp2-DTA $n=5$ cells, $n=2$ mice, $p>0.3$ by Wilcoxon rank sum test), and contrast response functions (wild type $n=6$ cells, $n=2$ mice, Pcp2-DTA $n=3$ cells, $n=2$ mice, $p>0.3$ by bootstrapping methods) were not significantly different between wild-type and Pcp2-DTA mice

can form multiple synapses with a single $\operatorname{rod}^{8}$. Similar observations had previously been made in cat and primate retinas ${ }^{59,60}$. Using in vivo electroporation and AAVs to label rods and RBCs, respectively, we analyzed the connectivity of a large number of these cells. Our findings confirm the diversity of rod-RBC synapse configurations, and give a more comprehensive account of their distribution (Fig. 1). In addition, we visualize dendritic interactions among neighboring RBCs. On average, we find that dendrites of adjacent RBCs overlap by $\sim 30 \%$ and share $\sim 13 \%$ of their input from rods (Fig. 2). Based on physiological evidence, Pang et al. ${ }^{61}$ suggested that the mouse retina may contain two distinct types of RBCs. Dendritic overlap and shared input among neighboring RBCs in our study form single continuous distributions (Fig. 2). Together with recent large-scale single cell expression profiling data ${ }^{62}$, this argues for a single RBC type, whose function may vary.

In Pcp2-DTA and Pax6-DTA mice, RBC dendrites expand in graded fashion (Fig. 4). Together with a previous study, which found an inverse relationship between RBC density and dendrite size across mouse strains ${ }^{33}$, our findings suggest that homotypic signals restrict dendrite growth of RBCs. At the border of Crenegative and Cre-positive regions in Pax6-DTA retinas, we find that dendritic growth of RBCs is directed away from remaining neighbors, suggesting that homotypic signals are local, possibly mediated by cell-cell contacts (Supplementary Fig. 4). The cell adhesion molecule DSCAM-LIKE 1 (DSCAML1) mediates repulsive interactions between $\mathrm{RBC}$ dendrites ${ }^{63}$. However, while DSCAML1 is required for self-avoidance, dendrite size is reduced, rather than increased, in Dscaml1 null mutants ${ }^{63}$. Thus, molecular identities of signals that control RBC dendrite size remain to be uncovered. The same or different signals may control RBC axon size, which increases in parallel with dendrite size in Pcp2DTA and Pax6-DTA mice (Fig. 6). In principle, local imbalances in activity introduced by removal of a fraction of RBCs could contribute to the changes in axon size. We think this is unlikely, because studies that silenced subsets of cone bipolar cells found axon territories to be unchanged ${ }^{14,47}$.
In addition to neurite territories, we find that cell densitydependent plasticity regulates synaptogenesis. As RBC dendrites expand in Pcp2-DTA and Pax6-DTA mice, they form fewer doublet and more singlet synapses with rods (Fig. 4). This shift in synapse configurations, in which the number of PSDs per presynaptic release site is adjusted, constitutes a novel plasticity mechanism. It is reminiscent of changes in multi-synaptic appositions in the inner retina ${ }^{16}$ and in same-dendrite multiple-synapse boutons in the hippocampus ${ }^{64}$. However, these architectures contain multiple presynaptic release sites, and were found to change during activity-dependent rather than cell density-dependent plasticity ${ }^{16,64}$.

We find that RBC dendrite and synapse development, and cell density-dependent plasticity are unchanged in a Gnat $1^{-/-}$background (Fig. 5). Similarly, a recent study found that clustering of mGluR6 receptors on $\mathrm{RBC}$ dendrites is not affected by dark rearing ${ }^{37}$. By contrast, rod-RBC synapses fail to form when vesicle fusion of rods is blocked by transgenic expression of tetanus toxin ${ }^{3}$. Thus, spontaneous rather than light-evoked signals from rods appear to shape the development of the rod bipolar pathway.

How different plasticity mechanisms of developing neurons are coordinated and to what end is not well understood. For RBC dendrites and axons in Pcp2-DTA and Pax6-DTA retinas, we find that adjustments of neurite territories are countered by opposite changes in synaptogenesis (Figs. 4, 6). Similar co-regulation of dendrite and synapse development was previously observed in Drosophila following perturbations of input activity ${ }^{65}$, indicating that coordinated plasticity may be an evolutionarily conserved feature of neural circuits. We propose that homeostatic coregulation of neurite growth and synaptogenesis serves to simultaneously optimize wiring of neuronal populations (i.e., input and target coverage) and individuals (i.e., input and output connectivity). In patch clamp recordings, we find that dim light responses of ON $\alpha-\mathrm{RGCs}$ and $\mathrm{OFF} \alpha-\mathrm{RGCs}$ in Pcp2-DTA mice are preserved, in spite of the $\sim 53 \%$ reduction in RBCs (Figs. 7, 8). This highlights the ability of homeostatically co-regulated plasticity mechanisms to stabilize circuit function. 
a
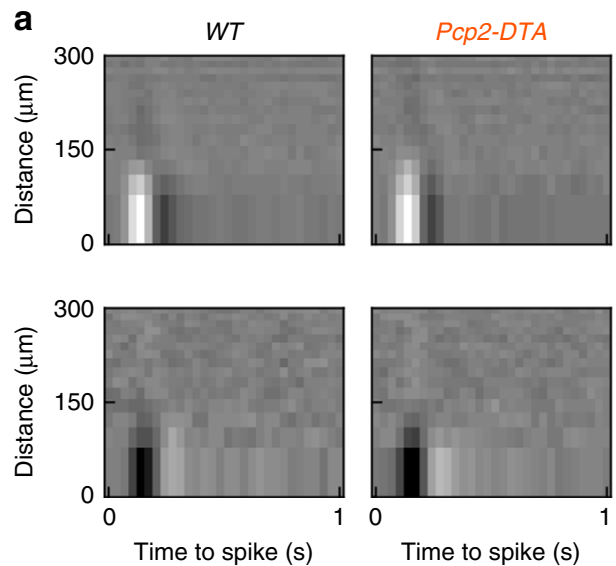

b
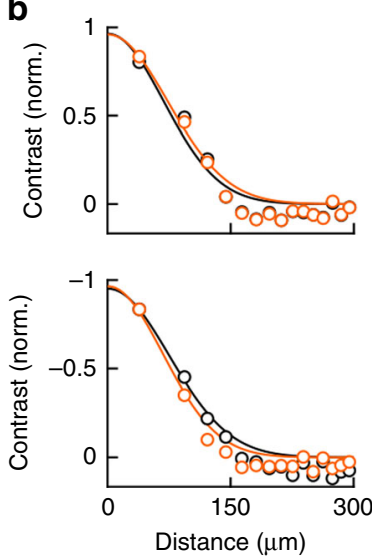

C
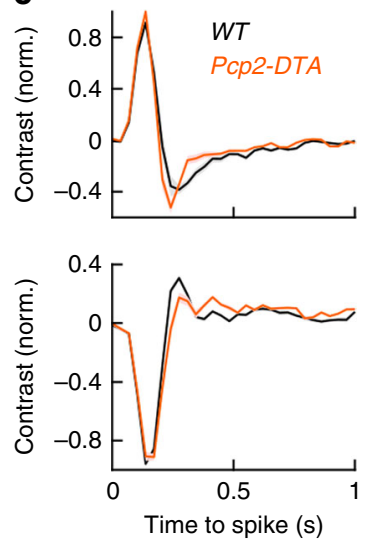

Fig. 8 Spatiotemporal receptive fields of ON $\alpha$-RGCs and OFF $\alpha$-RGCs in wild-type and Pcp2-DTA mice. a Representative spatiotemporal receptive field maps from ON $\alpha$-RGCs (top row) and OFF $\alpha$-RGCs (bottom row) in wild-type and Pcp2-DTA mice. Because the area of each ring in our circular white noise stimuli was kept constant, rows in the receptive field maps decrease in height with increasing distance from the center. b, c Summary data (mean \pm SEM) of the spatial and temporal response profiles at the temporal and spatial absolute response maxima, respectively (WT ON $\alpha$-RGC $n=12$ cells, WT OFF $\alpha$-RGCs $n=9, n=4$ mice; Pcp2-DTA ONa-RGC $n=6$, Pcp2-DTA OFF $\alpha$-RGC $n=7, n=3$ mice)

\section{Methods}

Mice. We generated mice in which a $9 \mathrm{~kb}$ fragment of the Grm6 promoter drives expression of YFP or, upon Cre-mediated recombination, of an attenuated version of diphtheria toxin $\left(G r m 6_{L^{-}} Y F P-D T A^{c o n}\right)^{16,47}$. To remove different numbers of RBCs from the developing retina, Grm6 ${ }_{L}-Y F P-D T A^{\text {con }}$ mice were crossed to Pax6$\mathrm{Cre}^{46}$ (RRID:MGI:4821787) and Pcp2-Cre ${ }^{45}$ (RRID: IMSR_JAX:010536) mice. We refer to double transgenic offspring from these crosses as Pax6-DTA and Pcp2-DTA mice, respectively. We tried to remove RBCs from the mature retina by crossing Pcp2-Cre mice, to a strain in which the diphtheria toxin receptor is expressed in a Cre-dependent manner (DTR mice, RRID:IMSR_JAX:007900 ${ }^{48}$. Double transgenic offspring from this cross $(P c p 2-D T R)$ was injected with diphtheria toxin $(1 \mu \mathrm{g} / 50 \mathrm{~g}$ body weight) intraperitoneally once every other day for a total of 4 days starting at $\mathrm{P}^{3} 0^{49}$. To evaluate the effect of light-evoked signals from rod photoreceptors on synaptic wiring and plasticity, Pax6-DTA mice were crossed to mice lacking rod transducin- $\alpha$ (Gnat $1^{-/-}$mice $)^{51}$. To block neurotransmitter release from rod photoreceptors, we crossed Rhodopsin-iCre ${ }^{54}$ mice to a strain in which the light chain of tetanus toxin is expressed in a Cre-dependent manner (TeNT mice $)^{52}$. In a subset of experiments, RBCs were labeled transgenically $\left(\mathrm{Grm}_{L^{-}}\right.$ tdTomato $)^{15}$. All mice were crossed onto a C57BL/6J background for more than five generations. Experiments were conducted using young adult mice (postnatal day 25 (P25)-P35) of both sexes. Mice were kept on a $12 \mathrm{~h}$ light/12 h dark cycle. For anatomy experiments, mice were typically killed in the morning after $2-4 \mathrm{~h}$ of light. For electrophysiology experiments, mice were dark-adapted overnight and killed in the subjective morning. The procedures in this study were approved by the Animal Studies Committee of Washington University School of Medicine and performed in compliance with the National Institutes of Health Guide for the Care and Use of Laboratory Animals.

Adeno-associated viruses. To label RBCs, we generated AAVs in which four concatenated repeats of a $200 \mathrm{bp}$ fragment of the Grm6 promoter ${ }^{42}$ drive expression of red $\left(\mathrm{Grm}_{\mathrm{S}}\right.$-tdTomato) or yellow fluorescent proteins $\left(\mathrm{Grm}_{S^{-}} \mathrm{YFP}\right)$. The $p A A V$ Grm $_{S^{-}}$YFP plasmid was created by switching the $C A G$ promoter of $p A A V-C A G-$ $Y F P^{5}$ with the Grm6 repeats using linkers containing Asp718I and EcoRI restriction sites introduced by PCR. $p A A V-G r m \sigma_{S^{-}}$tdTomato was then derived from $p A A V$ $G r m 6_{S}-Y F P$ by replacing YFP with tdTomato from a tdTomato-N1 vector (Addgene \#54642) using BamHI and NotI restriction sites. AAV1/2 chimeric virions were produced by co-transfecting HEK-293 cells with $p A A V-G r m 6_{S^{-}} Y F P$ or $p A A V$ Grm6 $6_{S}$-tdTomato, and helper plasmids encoding Rep2 and the Cap for serotype 1 and Rep2 and the Cap for serotype 2. Forty-eight hours after transfection, cells and supernatant were harvested and viral particles purified using heparin affinity columns (Sigma). Viruses $(250 \mathrm{~nL})$ were delivered into the vitreous chamber of newborn mice anesthetized on ice via a Nanoject II injector (Drummond).

In vivo electroporation. To label rod photoreceptors, we injected $p N r l-D s R e d$ plasmid $^{34}$ into the subretinal space of newborn mice anesthetized on ice via a Nanoject II injector (Drummond). To electroporate rods, five $80 \mathrm{~V}$ square pulses of $50 \mathrm{~ms}$ duration generated by an ECM830 (BTX Harvard Apparatus) were delivered via tweezer electrodes with the anode placed on the injected eye ${ }^{66}$.

Optic nerve crush. The optic nerve was exposed intraorbitally and crushed with forceps (Dumont \#55 FST) for $\sim 5 \mathrm{~s} \sim 1 \mathrm{~mm}$ behind the posterior surface of the eyeball.
Tissue preparation. Mice were killed with $\mathrm{CO}_{2}$ and enucleated. For vibratome sections, the cornea, lens, and vitreous were removed in in HEPES-buffered mouse

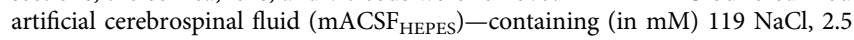
$\mathrm{KCl}, 2.5 \mathrm{CaCl}_{2}, 1.3 \mathrm{MgCl}_{2}, 1 \mathrm{NaH}_{2} \mathrm{PO}_{4}, 11$ glucose, and 20 HEPES (pH adjusted to 7.37 with $\mathrm{NaOH}$ ) - and the remaining eye cup fixed for $30 \mathrm{~min}$ in $4 \%$ paraf-

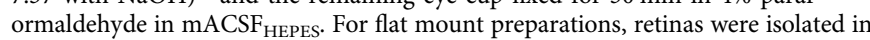
$\mathrm{mACSF}_{\text {HEPES}}$, mounted on membrane disks (HABGO1300, Millipore) and fixed for $30 \mathrm{~min}$ in $4 \%$ paraformaldehyde in $\mathrm{mACSF}_{\mathrm{HEPES}}$. For electrophysiology, retinas from dark-adapted mice $(>2 \mathrm{~h})$ were isolated under infrared illumination in bicarbonate-buffered mouse artificial cerebrospinal fluid ( $\mathrm{mACSF}_{\mathrm{NaHCO} 3}$ ) containing (in $\mathrm{mM}$ ) $125 \mathrm{NaCl}, 2.5 \mathrm{KCl}, 1 \mathrm{MgCl}_{2}, 1.25 \mathrm{NaH}_{2} \mathrm{PO}_{4}, 2 \mathrm{CaCl}_{2}, 20$ glucose, $26 \mathrm{NaHCO}_{3}$, and 0.5 L-Glutamine equilibrated with $95 \% \mathrm{O}_{2} / 5 \% \mathrm{CO}_{2}$ and flat mounted on transparent membrane discs (Anodisc, Whatman).

Immunohistochemistry. For tissue sections, retinas were isolated from fixed eye cups, embedded in $4 \%$ agarose and cut into $60 \mu \mathrm{m}$ slices on a vibratome (VT1000 A, Leica). Flat-mounted retinas were cryoprotected (10\% sucrose in phosphatebuffered saline (PBS) for $1 \mathrm{~h}$ at RT, $20 \%$ sucrose in PBS for $1 \mathrm{~h}$ at RT, and $30 \%$ sucrose in PBS overnight at $4^{\circ} \mathrm{C}$ ), frozen, and thawed three times and washed in PBS three times for $10 \mathrm{~min}$ at RT. Vibratome slices and flat mounts were then blocked in 5\% normal donkey serum (NDS) in PBS for $1 \mathrm{~h}$ at RT, before being incubated with primary antibodies in 5\% NDS and $0.5 \%$ Triton X-100 in PBS overnight (vibratome slices) or for 5 days (flat mounts) at $4^{\circ} \mathrm{C}$. The following primary antibodies were used in this study: mouse anti-CACNA1S to label Gpr179 ${ }^{38}$ (1:500, Millipore, RRID:AB_2069582), sheep anti-mGluR6 (1:200, Dr. K. Martemyanov $)^{67}$, mouse anti-CtBP2 (1:500, BD Biosciences, RRID:AB_399431), mouse anti-PKC $\alpha$ (1:1000, Sigma, RRID:AB_477375), rabbit anti-DsRed (1:1000, BD Biosciences, RRID:AB_394264), chicken anti-GFP (1:1000, ThermoFisher, RRID:AB_2534023). After incubation with primary antibodies, the tissue was washed in PBS three times for $10 \mathrm{~min}$ at RT, stained for $2 \mathrm{~h}$ at RT (vibratome slices) or overnight at $4{ }^{\circ} \mathrm{C}$ (flat mounts) with DyLight 405 (1:500, ThermoFisher, RRID: AB_2533208), Alexa 488 (1:1000, ThermoFisher, anti-chicken IgY, RRID: AB_2534096, anti-mouse IgG, RRID:AB_2534069), Alexa 568 (1:1000, ThermoFisher, anti-rabbit IgG, RRID:AB_143011), and Alexa 633 (1:1000, ThermoFisher, anti-mouse IgG RRID:AB_141459) secondary antibodies, washed again in PBS three times for $10 \mathrm{~min}$ at RT, and mounted in Vectashield medium (Vector Laboratories, RRID:AB_2336789).

Imaging and analysis. Confocal image stacks were acquired on an Olympus Fv1000 laser scanning microscope using a $60 \times 1.35$ NA oil immersion objective and a $20 \times 0.85$ NA oil immersion objective. Dendritic and axonal connectivity of $\mathrm{RBCs}$ was analyzed in image stacks with $0.066-0.3 \mu \mathrm{m}(x / y-z)$ voxels. Superresolution imaging (voxel size: $0.043-0.1 \mu \mathrm{m}, x / y-z$ ) was performed on a Zeiss LSM 880 microscope with an AiryScan detector array. To identify dendritic synapses of individual RBCs, we generated binary masks from the signal of fluorescent proteins expressed sparsely in RBCs and the signal of immunostaining for Gpr179 or mGluR6 using local thresholding in Amira (FEI). Receptor clusters at cone synapses occur lower in the outer plexiform layer and are morphologically clearly distinct from receptor clusters at rod synapses $3,37,68$. Clusters of immunostaining at rods were assigned to a given RBC if the respective masks overlapped. Dendritic territories were measured as the area of the smallest convex polygon to encompass synapses in a $z$-projection. To identify ribbon release sites of individual RBCs, their 
axons were masked by local thresholding in Amira. Axon masks were then applied to signals of immunostaining for the C-terminal binding protein 2 in the same image stack, and synaptic clusters detected using previously described algorithms ${ }^{14-16}$ implemented in MATLAB (The Mathworks, RRID:SCR_001622). Axon size was measured by the surface area of the binary mask.

\section{Electrophysiology and visual stimulation and analysis. Cell-attached and} whole-cell patch clamp recordings of ON $\alpha$-RGCs were obtained from the dorsal retina in flat mount preparations ${ }^{49,} 69$. Throughout the recordings, retinas were continually perfused $(5-7 \mathrm{~mL} / \mathrm{min})$ with warm $\left(\sim 33^{\circ} \mathrm{C}\right) \mathrm{mACSF}_{\mathrm{NaHCO}}$. The intracellular solution for current clamp recordings contained (in $\mathrm{mM}$ ) $125 \mathrm{~K}$ gluconate, $10 \mathrm{NaCl}, 1 \mathrm{MgCl}_{2}$, 10 EGTA, 5 HEPES, 5 ATP-Na, and 0.1 GTP-Na (pH adjusted to 7.2 with $\mathrm{KOH}$ ). The intracellular solution for voltage clamp recordings contained (in mM) 120 Cs-gluconate, $1 \mathrm{CaCl}_{2}, 1 \mathrm{MgCl}_{2}, 10 \mathrm{Na}$-HEPES, 11 EGTA, 10 TEA-Cl, and 2 Qx314 (pH adjusted to 7.2 with $\mathrm{CsOH}$ ). Patch pipettes had resistances of 4-7 M $\Omega$ (borosilicate glass). Signals were amplified with a Multiclamp 700B amplifier (Molecular Devices), filtered at $3 \mathrm{kHz}$ (8-pole Bessel lowpass) and sampled at $10 \mathrm{kHz}$ (Digidata $1440 \mathrm{~A}$, Molecular Devices). In voltage clamp recordings, series resistance (10-15 M $\Omega$ ) was compensated electronically by $\sim 75 \%$. Excitatory postsynaptic currents were isolated by holding cells at the reversal potential of inhibitory $(-60 \mathrm{mV})$ conductances. In current clamp recordings, no bias current was injected. ON $\alpha$-RGCs were selected under infrared illumination based on their large soma size (diameter $>20 \mu \mathrm{m}$ ); and correct targeting was confirmed by inclusion of Alexa 488 or Alexa $568(0.1 \mathrm{mM})$ in the intracellular solution and 2-photon imaging at the end of each recording.

Multielectrode array (MEA) recordings were obtained from rectangular $(\sim 1 \times$ $1.5 \mathrm{~mm}$ ) pieces of dorsal retina and were floated RGC side down onto an MEA (Multichannelsystems, 252 electrodes, $30 \mu \mathrm{m}$ electrode size, $100 \mu \mathrm{m}$ center-center spacing) secured by a transparent tissue culture membrane $(3 \mu \mathrm{m}$ pore size, Corning) weighed down by a platinum ring. Retinas were continually perfused $(5-7 \mathrm{~mL} / \mathrm{min})$ with warm $\left(\sim 33^{\circ} \mathrm{C}\right) \mathrm{mACSF}_{\mathrm{NaHCO}}$. Signals of each electrode were band-pass filtered between 300 and $3000 \mathrm{~Hz}$ and digitized at $10 \mathrm{kHz}$. Signal cut outs $(3 \mathrm{~ms})$ triggered on negative threshold crossings were written to hard disk together with the time of threshold crossing (i.e., spike time). Principal component analysis of these waveforms was used to sort spikes into trains representing the activity individual neurons (Offline Sorter, Plexon).

Visual stimuli were presented on an organic light-emitting display (OLED, eMagin) and projected onto the photoreceptor side of the retina via a substage condenser (patch clamp recordings) or through a $20 \times 0.5 \mathrm{NA}$ water immersion objective (MEA recordings). Photon fluxes at the preparation were calibrated with a photometer (UDT Instruments S471, 268R) and converted to photoisomerization rates based on the spectral output of the OLED measured with a Spectrometer (StellarNet, BLACK Comet), the rod spectral sensitivity, and a collecting area of $0.5 \mu \mathrm{m}^{270}$. Scotopic stimuli (mean intensity: 1.5 rhodopsin isomerization/rod/s, 1.5 $R^{*}$ ) were centered on the soma of the recorded cell. To test contrast sensitivity, short luminance steps $(250 \mathrm{~ms})$ were presented every $2.25 \mathrm{~s}$ in a circular area (diameter: $300 \mu \mathrm{m})^{57}$. Baseline-subtracted responses (spike rate or conductance) were measured during $100 \mathrm{~ms}$ time windows. Spatiotemporal receptive fields were analyzed by presenting circular white noise stimuli, in which the intensity of rings of equal area centered on the recorded cell was chosen at random every $33 \mathrm{~ms}$ (refresh rate: $30 \mathrm{~Hz}$ ) from a Gaussian distribution. Receptive field maps were then constructed by reverse correlation of the response with the stimulus via spiketriggered stimulus averaging ${ }^{57,58}$.

Electroretinograms. Responses to brief white light flashes $(<5 \mathrm{~ms})$ were acquired from Gnat $^{-{ }^{--}}$and littermate control mice (P30) using a UTAS Visual Electrodiagnostic Testing System (LKC Technologies). Dark-adapted mice were anesthetized with ketamine $(80 \mathrm{mg} / \mathrm{kg})$ and xylazine $(15 \mathrm{mg} / \mathrm{kg})$ and their pupils dilated with $1 \%$ atropine sulfate (Falcon Pharmaceuticals). Recording electrodes embedded in contact lenses were placed over the cornea of both eyes. At each light level 5-10 responses were averaged. The a-wave was measured as the difference between the response minimum in the first $50 \mathrm{~ms}$ after flash onset and the voltage value at flash onset; and the b-wave amplitude was measured as the difference between a 15-25 $\mathrm{Hz}$ low-pass-filtered b-wave peak and the a-wave amplitude. ERG analysis was performed using custom scripts written in MATLAB.

Statistics. Statistical significance of differences between morphological char acteristics (e.g., territory size, average number of synapses per rod) was assessed using Wilcoxon rank sum (for two groups) or Kruskal-Wallis one-way ANOVA (for more than two groups) tests. Contrast response functions were compared using bootstrapping. The summed squared difference between mean contrast response functions of ONa-RGCs in wild-type and Pcp2-DTA retinas was compared to the distribution of summed squared differences generated by randomly assigning recorded contrast response functions to the two genotypes.

Data availability. The data that support the findings of this study are available from the corresponding author upon reasonable request.
Received: 25 October 2016 Accepted: 8 September 2017

Published online: 31 October 2017

\section{References}

1. Field, G. D., Sampath, A. P. \& Rieke, F. Retinal processing near absolute threshold: from behavior to mechanism. Annu. Rev. Physiol. 67, 491-514 (2005).

2. Zeitz, C., Robson, A. G. \& Audo, I. Congenital stationary night blindness: an analysis and update of genotype-phenotype correlations and pathogenic mechanisms. Prog. Retin. Eye Res. 45, 58-110 (2015).

3. Cao, Y. et al. Mechanism for selective synaptic wiring of rod photoreceptors into the retinal circuitry and its role in vision. Neuron 87, 1248-1260 (2015).

4. Neuille, M. et al. LRIT3 is essential to localize TRPM1 to the dendritic tips of depolarizing bipolar cells and may play a role in cone synapse formation. Eur. J. Neurosci. 42, 1966-1975 (2015).

5. Soto, F., Watkins, K. L., Johnson, R. E., Schottler, F. \& Kerschensteiner, D. NGL-2 regulates pathway-specific neurite growth and lamination, synapse formation, and signal transmission in the retina. J. Neurosci. 33, 11949-11959 (2013).

6. Sato, S. et al. Pikachurin, a dystroglycan ligand, is essential for photoreceptor ribbon synapse formation. Nat. Neurosci. 11, 923-931 (2008).

7. Hoon, M., Okawa, H., Della Santina, L. \& Wong, R. O. Functional architecture of the retina: development and disease. Prog. Retin. Eye Res. 42, 44-84 (2014).

8. Tsukamoto, Y. \& Omi, N. Functional allocation of synaptic contacts in microcircuits from rods via rod bipolar to AII amacrine cells in the mouse retina. J. Comp. Neurol. 521, 3541-3555 (2013).

9. Ebert, D. H. \& Greenberg, M. E. Activity-dependent neuronal signalling and autism spectrum disorder. Nature 493, 327-337 (2013).

10. Ramocki, M. B. \& Zoghbi, H. Y. Failure of neuronal homeostasis results in common neuropsychiatric phenotypes. Nature 455, 912-918 (2008).

11. Wong, R. O. \& Ghosh, A. Activity-dependent regulation of dendritic growth and patterning. Nat. Rev. Neurosci. 3, 803-812 (2002).

12. Antonini, A., Fagiolini, M. \& Stryker, M. P. Anatomical correlates of functional plasticity in mouse visual cortex. J. Neurosci. 19, 4388-4406 (1999).

13. Hua, J. Y., Smear, M. C., Baier, H. \& Smith, S. J. Regulation of axon growth in vivo by activity-based competition. Nature 434, 1022-1026 (2005).

14. Johnson, R. E. \& Kerschensteiner, D. Retrograde plasticity and differential competition of bipolar cell dendrites and axons in the developing retina. Curr. Biol. 24, 2301-2306 (2014)

15. Kerschensteiner, D., Morgan, J. L., Parker, E. D., Lewis, R. M. \& Wong, R. O. Neurotransmission selectively regulates synapse formation in parallel circuits in vivo. Nature 460, 1016-1020 (2009).

16. Morgan, J. L., Soto, F., Wong, R. O. \& Kerschensteiner, D. Development of cell type-specific connectivity patterns of converging excitatory axons in the retina. Neuron 71, 1014-1021 (2011).

17. Misgeld, T. et al. Roles of neurotransmitter in synapse formation: development of neuromuscular junctions lacking choline acetyltransferase. Neuron 36, 635-648 (2002).

18. Burrone, J., O'Byrne, M. \& Murthy, V. N. Multiple forms of synaptic plasticity triggered by selective suppression of activity in individual neurons. Nature $\mathbf{4 2 0}$, 414-418 (2002).

19. Glebov, O. O., Cox, S., Humphreys, L. \& Burrone, J. Neuronal activity controls transsynaptic geometry. Sci. Rep. 6, 22703 (2016).

20. Wefelmeyer, W., Puhl, C. J. \& Burrone, J. Homeostatic plasticity of subcellular neuronal structures: from inputs to outputs. Trends Neurosci. 39, 656-667 (2016).

21. O'Brien, R. J. et al. Activity-dependent modulation of synaptic AMPA receptor accumulation. Neuron 21, 1067-1078 (1998).

22. Lohmann, C., Myhr, K. L. \& Wong, R. O. Transmitter-evoked local calcium release stabilizes developing dendrites. Nature 418, 177-181 (2002).

23. Harvey, C. D., Yasuda, R., Zhong, H. \& Svoboda, K. The spread of Ras activity triggered by activation of a single dendritic spine. Science 321, 136-140 (2008).

24. Lee, M. C., Yasuda, R. \& Ehlers, M. D. Metaplasticity at single glutamatergic synapses. Neuron 66, 859-870 (2010).

25. Buss, R. R., Sun, W. \& Oppenheim, R. W. Adaptive roles of programmed cell death during nervous system development. Annu. Rev. Neurosci. 29, 1-35 (2006).

26. Young, R. W. Cell death during differentiation of the retina in the mouse. J. Comp. Neurol. 229, 362-373 (1984).

27. Wassle, H. Parallel processing in the mammalian retina. Nat. Rev. Neurosci. 5 , 747-757 (2004).

28. Masland, R. H. The fundamental plan of the retina. Nat. Neurosci. 4, 877-886 (2001).

29. Huckfeldt, R. M. et al. Transient neurites of retinal horizontal cells exhibit columnar tiling via homotypic interactions. Nat. Neurosci. 12, 35-43 (2009). 
30. Reese, B. E. Development of the retina and optic pathway. Vision Res. 51, 613-632 (2011).

31. Keeley, P. W. \& Reese, B. E. Morphology of dopaminergic amacrine cells in the mouse retina: independence from homotypic interactions. J. Comp. Neurol. 518, 1220-1231 (2010).

32. Keeley, P. W., Whitney, I. E., Raven, M. A. \& Reese, B. E. Dendritic spread and functional coverage of starburst amacrine cells. J. Comp. Neurol. 505, 539-546 (2007).

33. Keeley, P. W. et al. Independent genomic control of neuronal number across retinal cell types. Dev. Cell 30, 103-109 (2014).

34. Matsuda, T. \& Cepko, C. L. Controlled expression of transgenes introduced by in vivo electroporation. Proc. Natl Acad. Sci. USA 104, 1027-1032 (2007).

35. Swaroop, A. et al. A conserved retina-specific gene encodes a basic motif/ leucine zipper domain. Proc. Natl Acad. Sci. USA 89, 266-270 (1992).

36. Tsukamoto, Y., Morigiwa, K., Ueda, M. \& Sterling, P. Microcircuits for night vision in mouse retina. J. Neurosci. 21, 8616-8623 (2001).

37. Dunn, F. A., Della Santina, L., Parker, E. D. \& Wong, R. O. Sensory experience shapes the development of the visual system's first synapse. Neuron $\mathbf{8 0}$, 1159-1166 (2013).

38. Hasan, N., Ray, T. A. \& Gregg, R. G. CACNA1S expression in mouse retina: novel isoforms and antibody cross-reactivity with GPR179. Vis. Neurosci. 33, E009 (2016).

39. Peachey, N. S. et al. GPR179 is required for depolarizing bipolar cell function and is mutated in autosomal-recessive complete congenital stationary night blindness. Am. J. Hum. Genet. 90, 331-339 (2012).

40. Audo, I. et al. Whole-exome sequencing identifies mutations in GPR179 leading to autosomal-recessive complete congenital stationary night blindness. Am. J. Hum. Genet. 90, 321-330 (2012).

41. Nomura, A. et al. Developmentally regulated postsynaptic localization of a metabotropic glutamate receptor in rat rod bipolar cells. Cell 77, 361-369 (1994).

42. Lagali, P. S. et al. Light-activated channels targeted to ON bipolar cells restore visual function in retinal degeneration. Nat. Neurosci. 11, 667-675 (2008).

43. Wassle, H., Puller, C., Muller, F. \& Haverkamp, S. Cone contacts, mosaics, and territories of bipolar cells in the mouse retina. J. Neurosci. 29, 106-117 (2009).

44. Breitman, M. L., Rombola, H., Maxwell, I. H., Klintworth, G. K. \& Bernstein, A. Genetic ablation in transgenic mice with an attenuated diphtheria toxin A gene. Mol. Cell. Biol. 10, 474-479 (1990).

45. Zhang, X. M. et al. Highly restricted expression of Cre recombinase in cerebellar Purkinje cells. Genesis 40, 45-51 (2004).

46. Marquardt, T. et al. Pax6 is required for the multipotent state of retinal progenitor cells. Cell 105, 43-55 (2001).

47. Okawa, H., Della Santina, L., Schwartz, G. W., Rieke, F. \& Wong, R. O. Interplay of cell-autonomous and nonautonomous mechanisms tailors synaptic connectivity of converging axons in vivo. Neuron 82, 125-137 (2014).

48. Buch, T. et al. A Cre-inducible diphtheria toxin receptor mediates cell lineage ablation after toxin administration. Nat. Methods 2, 419-426 (2005).

49. Tien, N. W., Kim, T. \& Kerschensteiner, D. Target-specific glycinergic transmission from VGluT3-expressing amacrine cells shapes suppressive contrast responses in the retina. Cell Rep. 15, 1369-1375 (2016).

50. Krishnaswamy, A., Yamagata, M., Duan, X., Hong, Y. K. \& Sanes, J. R. Sidekick 2 directs formation of a retinal circuit that detects differential motion. Nature 524, 466-470 (2015).

51. Calvert, P. D. et al. Phototransduction in transgenic mice after targeted deletion of the rod transducin alpha -subunit. Proc. Natl Acad. Sci. USA 97, 13913-13918 (2000)

52. Kim, J. C. et al. Linking genetically defined neurons to behavior through a broadly applicable silencing allele. Neuron 63, 305-315 (2009).

53. Shimshek, D. R. et al. Codon-improved Cre recombinase (iCre) expression in the mouse. Genesis 32, 19-26 (2002).

54. Li, S. et al. Rhodopsin-iCre transgenic mouse line for Cre-mediated rod-specific gene targeting. Genesis 41, 73-80 (2005).

55. Schmitz, F., Konigstorfer, A. \& Sudhof, T. C. RIBEYE, a component of synaptic ribbons: a protein's journey through evolution provides insight into synaptic ribbon function. Neuron 28, 857-872 (2000).

56. Grimes, W. N., Schwartz, G. W. \& Rieke, F. The synaptic and circuit mechanisms underlying a change in spatial encoding in the retina. Neuron $\mathbf{8 2}$, 460-473 (2014).

57. Pearson, J. T. \& Kerschensteiner, D. Ambient illumination switches contrast preference of specific retinal processing streams. J. Neurophysiol. 114, 540-550 (2015).

58. Chichilnisky, E. J. A simple white noise analysis of neuronal light responses. Network 12, 199-213 (2001).

59. Grunert, U. \& Martin, P. R. Rod bipolar cells in the macaque monkey retina: immunoreactivity and connectivity. J. Neurosci. 11, 2742-2758 (1991).

60. Sterling, P., Freed, M. A. \& Smith, R. G. Architecture of rod and cone circuits to the on-beta ganglion cell. J. Neurosci. 8, 623-642 (1988).
61. Pang, J. J. et al. Direct rod input to cone BCs and direct cone input to rod BCs challenge the traditional view of mammalian BC circuitry. Proc. Natl Acad. Sci. USA 107, 395-400 (2010).

62. Shekhar, K., et al. Comprehensive classification of retinal bipolar neurons by single-cell transcriptomics. Cell 166, 1308-1323.e1330 (2016).

63. Fuerst, P. G. et al. DSCAM and DSCAML1 function in self-avoidance in multiple cell types in the developing mouse retina. Neuron 64, 484-497 (2009).

64. Yuste, R. \& Bonhoeffer, T. Morphological changes in dendritic spines associated with long-term synaptic plasticity. Annu. Rev. Neurosci. 24, 1071-1089 (2001).

65. Tripodi, M., Evers, J. F., Mauss, A., Bate, M. \& Landgraf, M. Structural homeostasis: compensatory adjustments of dendritic arbor geometry in response to variations of synaptic input. PLoS Biol. 6, e260 (2008).

66. Matsuda, T. \& Cepko, C. L. Analysis of gene function in the retina. Methods Mol. Biol. 423, 259-278 (2008).

67. Cao, Y. et al. Retina-specific GTPase accelerator RGS11/G beta 5S/R9AP is a constitutive heterotrimer selectively targeted to mGluR6 in ON-bipolar neurons. J. Neurosci. 29, 9301-9313 (2009).

68. Wang, Y. et al. The auxiliary calcium channel subunit alpha2delta4 is required for axonal elaboration, synaptic transmission, and wiring of rod photoreceptors. Neuron 93, 1359-1374 (2017).

69. Kim, T., Soto, F., Kerschensteiner, D. An excitatory amacrine cell detects object motion and provides feature-selective input to ganglion cells in the mouse retina. Elife 4, e08025 (2015).

70. Field, G. D. \& Rieke, F. Nonlinear signal transfer from mouse rods to bipolar cells and implications for visual sensitivity. Neuron 34, 773-785 (2002).

\section{Acknowledgements}

The promoter construct for $G r m 6_{L}-Y F P-D T A^{c o n}$ and $G r m 6_{L}$-tdTomato mice was a kind gift from Dr. S. Naganishi. Grm6 $\sigma_{L}-Y F P-D T A^{c o n}$ and $G r m 6_{L}-t d T o m a t o$ mice were generated by D.K. and R. Lewis in the laboratory of Dr. R.O.L. Wong. The promoter construct for $\mathrm{Grm}_{s^{-}}$tdTomato and $\mathrm{Grm} 6_{s^{-}}$YFP AAVs was generously provided by Dr. B. Roska. We are grateful to Drs. C. Cepko and J. Corbo for the Nrl-DsRed construct, Dr. K. Martemyanov for the mGluR6 antibody, Dr. S. Dymecki for TeNT mice, and Dr. C.-K. Chen for Rhodopsin-iCre mice. We thank L. Zhao for expert technical assistance, Dr. P. Williams for help with the optic nerve crush experiment, and Dr. R. Apte and A. Santeford for advice and materials for staining microglia. This work was supported by the National Institutes of Health (EY023341 and EY027411 to

D.K., EY026978 to F.S. and D.K., and the Vision Core Grant EY0268) and the Research to Prevent Blindness Foundation (Career Development Award to D.K., and an unrestricted grant to the Department of Ophthalmology and Visual Sciences at Washington University).

\section{Author contribution}

R.E.J., N.-W.T., N.S., J.T.P., F.S., and D.K. designed, performed, and analyzed the experiments. R.E.J. and D.K. conceived the study and wrote the manuscript with input from all authors.

\section{Additional information}

Supplementary Information accompanies this paper at doi:10.1038/s41467-017-01332-7.

Competing interests: The authors declare no competing financial interests.

Reprints and permission information is available online at http://npg.nature.com/ reprintsandpermissions/

Publisher's note: Springer Nature remains neutral with regard to jurisdictional claims in published maps and institutional affiliations.

\footnotetext{
Open Access This article is licensed under a Creative Commons AY
adtribution 4.0 International License, which permits use, sharing, appropriate credit to the original author(s) and the source, provide a link to the Creative Commons license, and indicate if changes were made. The images or other third party material in this article are included in the article's Creative Commons license, unless indicated otherwise in a credit line to the material. If material is not included in the article's Creative Commons license and your intended use is not permitted by statutory regulation or exceeds the permitted use, you will need to obtain permission directly from the copyright holder. To view a copy of this license, visit http://creativecommons.org/ licenses/by/4.0/.
}

(C) The Author(s) 2017 